\title{
The quiescent $\mathrm{H}$-mode regime for high performance edge localized mode-stable operation in future burning plasmas
}

Citation for published version (APA):

Garofalo, A. M., Burrell, K. H., Eldon, D., Grierson, B. A., Hanson, J. M., Holland, C., Huijsmans, G. T. A., Liu, F., Loarte, A., Meneghini, O., Osborne, T. H., Paz-Soldan, C., Smith, S. P., Snyder, P. B., Solomon, W. M., Turnbull, A. D., \& Zeng, L. (2015). The quiescent $\mathrm{H}$-mode regime for high performance edge localized modestable operation in future burning plasmas. Physics of Plasmas, 22, 056116-1/12.

https://doi.org/10.1063/1.4921406

DOI:

$10.1063 / 1.4921406$

Document status and date:

Published: 01/01/2015

Document Version:

Publisher's PDF, also known as Version of Record (includes final page, issue and volume numbers)

Please check the document version of this publication:

- A submitted manuscript is the version of the article upon submission and before peer-review. There can be important differences between the submitted version and the official published version of record. People interested in the research are advised to contact the author for the final version of the publication, or visit the $\mathrm{DOI}$ to the publisher's website.

- The final author version and the galley proof are versions of the publication after peer review.

- The final published version features the final layout of the paper including the volume, issue and page numbers.

Link to publication

\section{General rights}

Copyright and moral rights for the publications made accessible in the public portal are retained by the authors and/or other copyright owners and it is a condition of accessing publications that users recognise and abide by the legal requirements associated with these rights.

- Users may download and print one copy of any publication from the public portal for the purpose of private study or research.

- You may not further distribute the material or use it for any profit-making activity or commercial gain

- You may freely distribute the URL identifying the publication in the public portal.

If the publication is distributed under the terms of Article 25fa of the Dutch Copyright Act, indicated by the "Taverne" license above, please follow below link for the End User Agreement:

www.tue.nl/taverne

Take down policy

If you believe that this document breaches copyright please contact us at:

openaccess@tue.nl

providing details and we will investigate your claim. 


\section{AIP $\mid \begin{aligned} & \text { Physics of } \\ & \text { Plasmas }\end{aligned}$}

\section{The quiescent $\mathrm{H}$-mode regime for high performance edge localized mode-stable operation in future burning plasmasa)}

A. M. Garofalo, K. H. Burrell, D. Eldon, B. A. Grierson, J. M. Hanson, C. Holland, G. T. A. Huijsmans, F. Liu, A. Loarte, O. Meneghini, T. H. Osborne, C. Paz-Soldan, S. P. Smith, P. B. Snyder, W. M. Solomon, A. D. Turnbull, and L. Zeng

Citation: Physics of Plasmas 22, 056116 (2015); doi: 10.1063/1.4921406

View online: http://dx.doi.org/10.1063/1.4921406

View Table of Contents: http://scitation.aip.org/content/aip/journal/pop/22/5?ver=pdfcov

Published by the AIP Publishing

\section{Articles you may be interested in}

Edge-localized mode avoidance and pedestal structure in I-mode plasmasa)

Phys. Plasmas 21, 056103 (2014); 10.1063/1.4872220

Global gyrokinetic simulations of the H-mode tokamak edge pedestala)

Phys. Plasmas 20, 055902 (2013); 10.1063/1.4803890

Shear flows at the tokamak edge and their interaction with edge-localized modesa)

Phys. Plasmas 14, 056118 (2007); 10.1063/1.2727330

Interplay between ballooning and peeling modes in simulations of the time evolution of edge localized modes Phys. Plasmas 12, 012506 (2005); 10.1063/1.1832600

Stability analysis of $\mathrm{H}$-mode pedestal and edge localized modes in a Joint European Torus power scan Phys. Plasmas 11, 1469 (2004); 10.1063/1.1668646

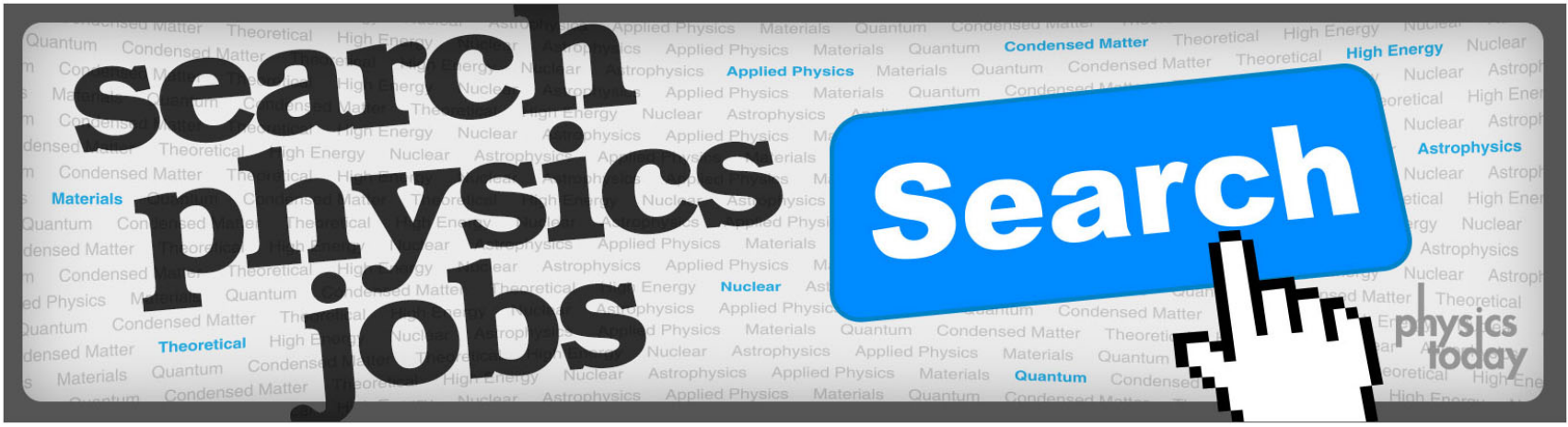




\title{
The quiescent $\mathrm{H}$-mode regime for high performance edge localized mode-stable operation in future burning plasmas ${ }^{\text {a) }}$
}

\author{
A. M. Garofalo, ${ }^{1, b), c)}$ K. H. Burrell, ${ }^{1}$ D. Eldon, ${ }^{2}$ B. A. Grierson, ${ }^{2}$ J. M. Hanson, ${ }^{3}$ C. Holland, ${ }^{4}$ \\ G. T. A. Huijsmans,${ }^{5}$ F. Liu ${ }^{5}$ A. Loarte,${ }^{5}$ O. Meneghini, ${ }^{1}$ T. H. Osborne,${ }^{1}$ C. Paz-Soldan, ${ }^{1}$ \\ S. P. Smith, ${ }^{1}$ P. B. Snyder, ${ }^{1}$ W. M. Solomon, ${ }^{2}$ A. D. Turnbull, ${ }^{1}$ and L. Zeng ${ }^{6}$ \\ ${ }^{1}$ General Atomics, P.O. Box 85608, San Diego, California 92186-5608, USA \\ ${ }^{2}$ Princeton Plasma Physics Laboratory, P.O. Box 451, Princeton, New Jersey 08543-0451, USA \\ ${ }^{3}$ Columbia University, 2960 Broadway, New York, New York 10027-6900, USA \\ ${ }^{4}$ University of California San Diego, 9500 Gilman Dr., La Jolla, California 92093-0417, USA \\ ${ }^{5}$ ITER Organization, Route de Vinon sur Verdon, 13067 St Paul Lez Durance, France \\ ${ }^{6}$ University of California Los Angeles, P.O. Box 957099, Los Angeles, California 90095-7099, USA
}

(Received 26 March 2015; accepted 8 May 2015; published online 26 May 2015)

For the first time, DIII-D experiments have achieved stationary quiescent $\mathrm{H}$-mode (QH-mode) operation for many energy confinement times at simultaneous ITER-relevant values of beta, confinement, and safety factor, in an ITER-like shape. QH-mode provides excellent energy confinement, even at very low plasma rotation, while operating without edge localized modes (ELMs) and with strong impurity transport via the benign edge harmonic oscillation (EHO). By tailoring the plasma shape to improve the edge stability, the QH-mode operating space has also been extended to densities exceeding $80 \%$ of the Greenwald limit, overcoming the long-standing low-density limit of QH-mode operation. In the theory, the density range over which the plasma encounters the kinkpeeling boundary widens as the plasma cross-section shaping is increased, thus increasing the $\mathrm{QH}-$ mode density threshold. The DIII-D results are in excellent agreement with these predictions, and nonlinear magnetohydrodynamic analysis of reconstructed $\mathrm{QH}$-mode equilibria shows unstable low n kink-peeling modes growing to a saturated level, consistent with the theoretical picture of the EHO. Furthermore, high density operation in the QH-mode regime has opened a path to a new, previously predicted region of parameter space, named "Super H-mode" because it is characterized by very high pedestals that can be more than a factor of two above the peeling-ballooning stability limit for similar ELMing H-mode discharges at the same density. (C) 2015 AIP Publishing LLC.

[http://dx.doi.org/10.1063/1.4921406]

\section{INTRODUCTION}

The quiescent H-mode (QH-mode) edge regime ${ }^{1}$ addresses several requirements for operation in $\operatorname{ITER}^{2}$ or a reactor: it avoids the pulsed edge localized mode (ELM) power fluxes to the plasma facing components (PFCs), the role of the ELMs for edge particle transport being replaced, usually, by an "edge harmonic oscillation" (EHO); it operates at ITER's values of collisionality and beta; it enables excellent energy confinement quality, necessary for high fusion performance, even at the very low plasma rotation expected for ITER; it improves the plasma stability by avoiding ELM-triggering of tearing modes, which is particularly severe at the relatively low rotation expected for ITER. The discharge in Fig. 1 illustrates several of these features.

However, extrapolation of the QH-mode to ITER or other burning plasma experiments is still uncertain, since the physics basis of QH-mode is not fully developed and validated. This paper discusses several recent experimental and modeling results that have increased our confidence in achieving QHmode for high confinement, ELM-stable operation in ITER: For the first time, $\mathrm{QH}$-mode has been achieved and maintained

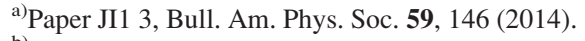

${ }^{\mathrm{b})}$ Invited speaker.

${ }^{c)}$ Author to whom correspondence should be addressed. Electronic mail: garofalo@fusion.gat.com
}

in an ITER-like cross section shape at a level of normalized fusion performance correspondent to $Q=10$ in the ITER Baseline scenario. Also for the first time, QH-mode operation has been extended to ITER-relevant values of normalized density. This has led to the discovery of an edge regime previously predicted by peeling-ballooning (P-B) theory, called Super Hmode, because it is characterized by about twice the edge pedestal pressure possible in standard $\mathrm{H}$-mode at high pedestal density. Thus, these experiments have provided new benchmarking of the P-B theory. In addition to successfully predicting access to Super H-mode, the theory successfully predicts the dependence of QH-mode density thresholds on the plasma cross-section shaping. Applied to ITER, this theory predicts that the ITER edge will operate on the pedestal stability boundary where QH-mode can exist. In a complementary effort, nonlinear magnetohydrodynamic (MHD) simulations confirm the hypothesis that the EHO consists of saturated kink-peeling modes. The nonlinear modeling and new measurements have also yielded the first evidence that these saturated modes lead to an ergodic layer at the plasma edge, just inside the separatrix. These results are essential steps on the path to predictive modeling of the EHO and its effect on the edge particle transport. Finally, recent experiments have measured impurity transport rates during $\mathrm{QH}$-mode operation and show that $\mathrm{QH}-$ mode particle transport is fast enough for impurity exhaust without ELMs. 

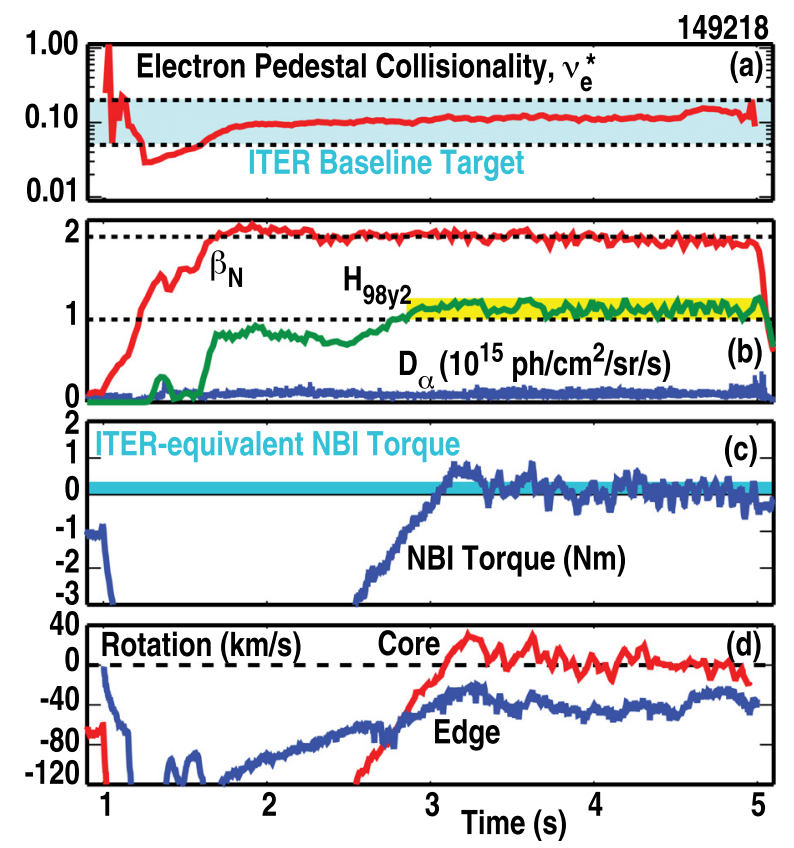

FIG. 1. Example QH-mode discharge with edge safety factor $q_{95} \sim 4.5$ operating without ELMs through a ramp down of the injected neutral beam torque from high values in the counter- $I_{\mathrm{p}}$ direction to near zero or slightly co- $I_{\mathrm{p}}$ (ITER relevant). Time traces of the (a) pedestal collisionality; (b) normalized beta, confinement improvement factor $\mathrm{H}_{98 \mathrm{y} 2}$ relative to ITER $\mathrm{H}-$ mode scaling, and $\mathrm{D}_{\alpha}$ light showing that bursting behavior from ELMs is absent; (c) torque from NBI; (d) toroidal rotation in the plasma core ( $\rho \sim 0.1$ ) and edge ( $\rho \sim 0.8$ ), as measured by charge exchange recombination spectroscopy using emission from carbon impurity ions. Negative torque and rotation are in direction counter to the plasma current. Edge counter rotation is driven by externally applied static, non-axisymmetric, nonresonant magnetic fields.

The paper is organized as follows. The next section (Sec. II) discusses the development of QH-mode operation for ITER Baseline target performance $(Q=10)$, including low rotation. Section III discusses the development of QHmode operation at high density, both absolute and normalized. Section IV reviews progress in developing a predictive understanding of $\mathrm{QH}$-mode for operations in future burning plasma devices, in particular, ITER. Section V briefly discusses QH-mode impurity transport. Section VI presents the summary and conclusions.

\section{DEVELOPMENT OF QH-MODE OPERATION FOR ITER BASELINE TARGET PERFORMANCE $(Q=10)$, INCLUDING LOW ROTATION}

At high rotation, robust $\mathrm{QH}-$ mode edge operation can be obtained in an ITER similar shape and at ITER baseline parameters of $\beta_{\mathrm{N}}, \mathrm{H}_{98 \mathrm{y} 2}$, and $q_{95} \cdot \beta_{\mathrm{N}}=\beta /\left(I_{\mathrm{p}} / a B\right)$ is the normalized $\beta$, where $\beta=\langle\mathrm{p}\rangle /\left(B^{2} / 2 \mu_{\mathrm{o}}\right)$ is the dimensionless plasma pressure, $I_{\mathrm{p}}$ the toroidal plasma current, $a$ the plasma minor radius, and $B$ the magnetic field strength. $\mathrm{H}_{98 \mathrm{y} 2}$ is the confinement enhancement factor with respect to the ITER9 y82 H-mode confinement scaling, ${ }^{3}$ and $q_{95}$ is the value of the safety factor, $q$, at the $95 \%$ flux surface. An example QH-mode discharge with normalized fusion performance equivalent to $Q=10$ in ITER is shown in Fig. 2, compared to a standard ITER baseline scenario discharge with nearly same toroidal field $(\sim 1.9 \mathrm{~T})$. The $\mathrm{QH}$-mode discharge uses strong neutral beam injection (NBI) torque in the direction
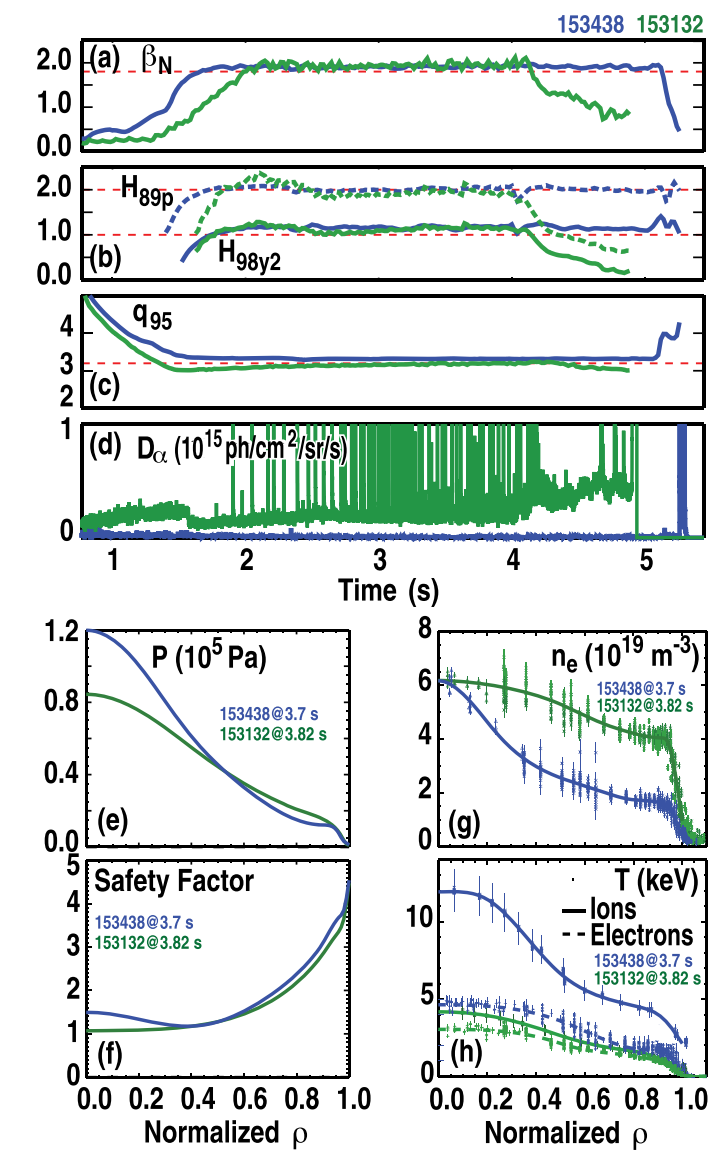

FIG. 2. Comparison of QH-mode (blue) and ELMing (green) H-mode discharges operating at ITER Baseline parameters of (a) normalized beta; (b) confinement factor $\mathrm{H}_{98 \mathrm{y} 2}$; and (c) edge safety factor. $\mathrm{D}_{\alpha}$ light in (d) shows the presence or absence of ELMs. Panels (e), (f), (g), and (h) compare the radial profiles of pressure, safety factor, electron density, ion, and electron temperature. Both discharges are obtained in an ITER-like single-null divertor cross-section shape.

counter to the plasma current (counter- $I_{\mathrm{p}} \mathrm{NBI}$ ). The standard scenario uses strong co $I_{\mathrm{p}} \mathrm{NBI}$ torque, and has ELMs. It is useful to look at the differences in the profiles. Because of the plasma formation using strong counter- $I_{\mathrm{p}} \mathrm{NBI}$, the $\mathrm{QH}$ mode scenario normally exhibits a $q$-profile with negative central magnetic shear (NCS) and $q_{\text {min }} \geq 1$, while a monotonic $q$-profile with $q_{\min } \sim 1$ (sawtoothing) is obtained in the standard scenario using co- $I_{\mathrm{p}} \mathrm{NBI}$ torque. Also, density is much lower in the $\mathrm{QH}$-mode scenario, but the temperature is much higher, yielding the same beta but much lower (and ITER similar) collisionality in QH-mode. Low collisionality may be implicated ${ }^{4}$ in the strong pressure peaking of these $\mathrm{QH}$-mode discharges. Note that the same pedestal height is obtained in QH mode and ELMing scenarios.

At low rotation, plasma operation at ITER baseline parameters of $\beta_{\mathrm{N}}, \mathrm{H}_{98 \mathrm{y} 2}$, and $q_{95}$ is quite challenging. As mentioned in the Introduction, the rotation in ITER is expected to be quite low. The plot in Fig. 3 shows the rotation predicted for ITER ${ }^{5}$ compared to the rotation in the discharges of Fig. 2. The ITER rotation is about an order of magnitude slower. Note, in addition, that this rotation is low compared to normal operation in any other present day machine, with or without NBI. The rotation predicted for ITER is very low despite the large NBI torque $(\sim 35 \mathrm{Nm})$, when compared to present machines, because the moment of inertia is expected to 
Toroidal Rotation (krad/s)

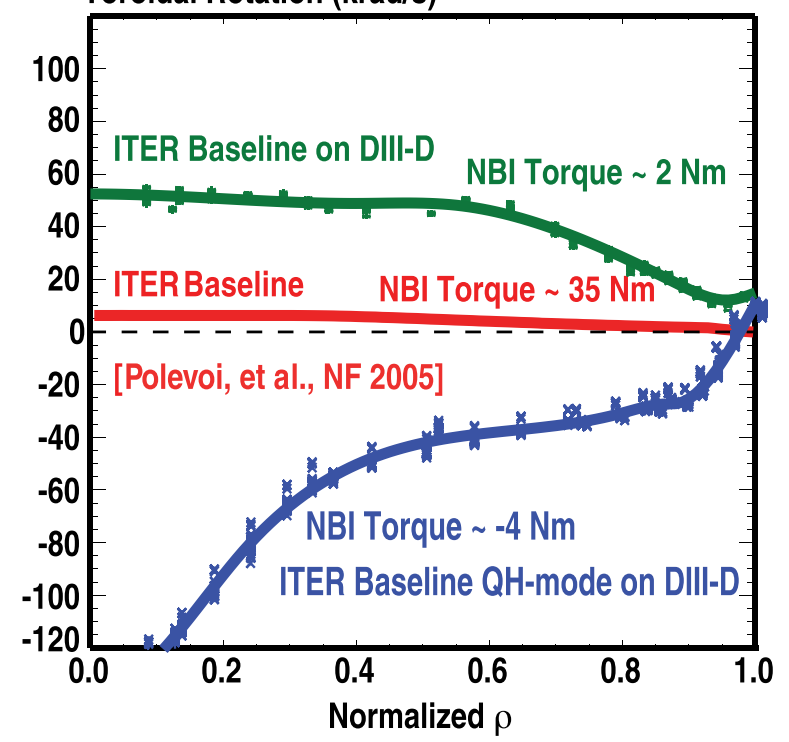

FIG. 3. Rotation profiles for typical values of the NBI torque in ELMing and QH-mode versions of the ITER baseline scenario in DIII-D, compared to the predicted rotation in the actual ITER baseline scenario.

increase with machine size much more rapidly than the angular momentum confinement time. In Ref. 6, we estimated that the equivalent NBI torque on DIII-D to generate an ITER-like rotation is $\sim 0.5 \mathrm{Nm}$ in the co- $I_{\mathrm{p}}$ direction. When on DIII-D we reduce the torque to approach this ITER-equivalent value, the plasma duration is usually limited by locked modes in both ITER baseline scenario versions: standard ELMing and QHmode. In recent work, the standard scenario has made good progress toward reproducible ELMing discharges at lower torque. $^{7}$ However, this progress is so far limited to operation at high collisionality above the ITER target range.

The challenge of $\mathrm{QH}$-mode operation at low NBI torque and plasma rotation is peculiar to the condition with parameters of $\beta_{\mathrm{N}}, \mathrm{H}_{98 \mathrm{y} 2}$, and $q_{95}$ simultaneously matching the ITER Baseline targets. At lower $\beta_{\mathrm{N}}(\sim 1.6)$ and higher $q_{95}(\sim 5.5)$, $\mathrm{QH}$-mode edge has been maintained with NBI torque smoothly going from counter- $I_{\mathrm{p}}$ to co- $I_{\mathrm{p}}$, as shown in Fig. 4. Note how the coherent EHO disappears at small rotation and only broadband MHD remains. Still, broadband MHD provides sufficient edge transport to avoid ELMs as the discharge goes through zero rotation.

At the ITER Baseline $\beta_{\mathrm{N}}$ value of $\sim 2$, but still higher $q_{95}(>4)$ than ITER Baseline, QH-mode edge also has been maintained with NBI torque smoothly going from counter- $I_{\mathrm{p}}$ to co- $I_{\mathrm{p}}$, as shown in Fig. 1, but only with the help of the counter- $I_{\mathrm{p}}$ torque driven by static $n=3$ nonresonant magnetic fields (NRMFs), which on DIII-D can be applied using non-axisymmetric coils internal to the vessel (I-coils) ${ }^{6}$ or external (C-coil). ${ }^{8,9}$ As $q_{95}$ is reduced toward ITER Baseline value of $\sim 3$, higher NBI torque is required to avoid $n=1$ locked modes and maintain stable operation, as shown by the experiments illustrated in Fig. 5. In these QH-mode discharges with similar $\beta_{\mathrm{N}}$ but different $q_{95}$, the NBI torque magnitude is ramped down toward zero, while an $n=3$ NRMF is applied. At $q_{95} \sim 3.3$, a threshold NBI torque of magnitude $\sim 4.5 \mathrm{Nm}$ is observed, at which point an $n=1$ locked mode terminates the high beta phase.
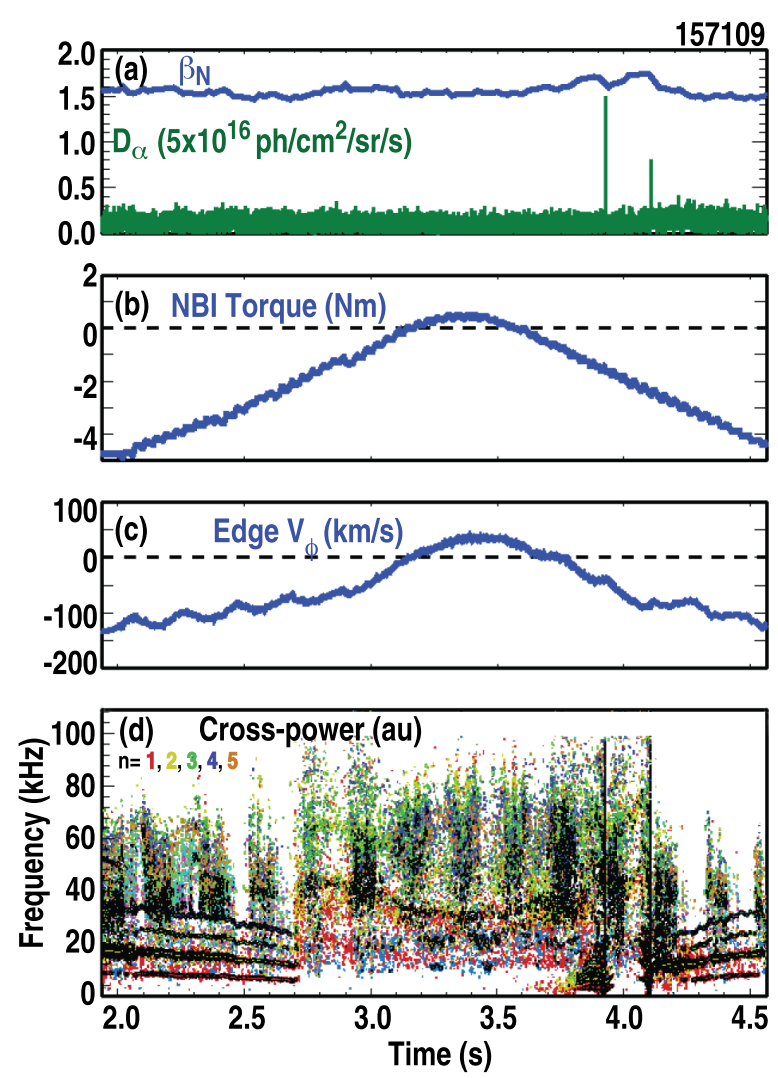

FIG. 4. Example of QH-mode operation at very low NBI torque without nonresonant magnetic fields. Time traces of the (a) normalized beta and $\mathrm{D}_{\alpha}$ light; (b) NBI torque; (c) edge toroidal rotation $(\rho \sim 0.8)$. Panel (d) shows the spectrum of magnetic fluctuations versus frequency and time, color coded for toroidal mode number, $n$.

In an effort to address the $n=1$ locked mode problem, recent experiments have extended the use of dynamic error field correction (DEFC) ${ }^{10}$ to the relatively low values of $\beta_{\mathrm{N}}$ of the ITER Baseline scenario. DEFC is the technique of maintaining tokamak axisymmetry by exploiting the changing plasma response to error fields, using a feedback system with magnetic sensors to control non-axisymmetric compensation coils. In previous successful uses, this technique exploited the strong increase in plasma response observed when the plasma beta increases from below to above the no-

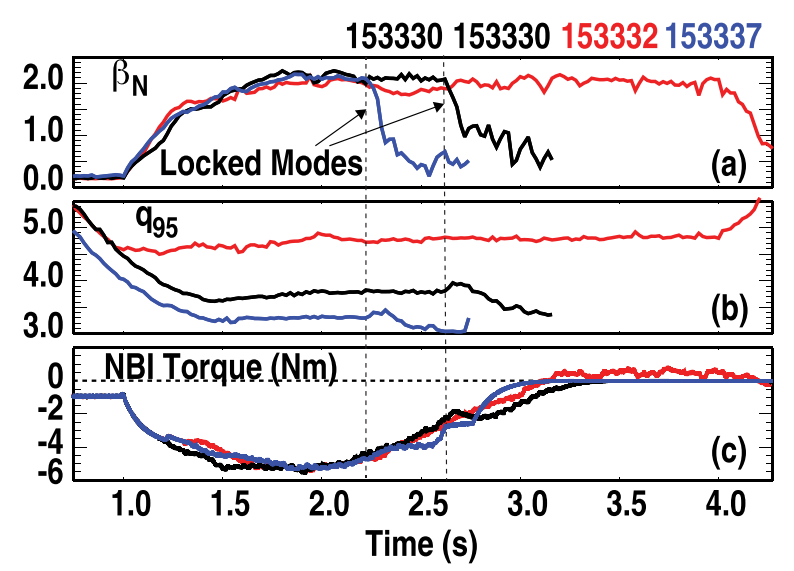

FIG. 5. NBI torque ramp experiments in similar discharges with different edge safety factor, $q_{95}$. Time traces of the (a) normalized beta; (b) edge safety factor; (c) NBI torque. $n=1$ locked modes (onset marked by vertical dashed lines) become a problem at low NBI torque magnitude when $q_{95}<4$. 
wall MHD stability limit. With $\beta_{\mathrm{N}}$ below the no-wall limit in the ITER Baseline scenario experiments, DEFC can utilize the significant change in plasma response observed across the transition from low power L-mode confinement phase to higher power $\mathrm{H}$-mode confinement phase.

Figure 6 shows that a static $n=1$ magnetic field is observed to grow when $\beta_{\mathrm{N}}$ increases from $\leq 0.5$ in the Lmode phase to $\sim 2$ in the $\mathrm{H}$-mode phase. This $n=1 \mathrm{mag}$ netic field is interpreted as plasma amplification of uncorrected $n=1$ error fields, in this case mostly due to an $n=1$ sideband of the $n=3$ field from the C-coil. The sideband is small (3.6\% of the $n=3$ field amplitude, giving $\sim 4$ Gauss of $n=1$ radial field at the outboard midplane in this case), but is amplified significantly by the plasma, even at the moderate beta of the ITER Baseline scenario. When the feedback system is turned on (just before the L-H transition and after the plasma current has reached flattop), the sensor signals are reset. From this point on, the error field is mostly unchanged, so only changes in the plasma response to the seed error field are measured. However, as it aims to reduce the plasma response, DEFC actually corrects the seed error field. Using a large feedback gain, only two discharge iterations were necessary for DEFC to nearly eliminate the $n=1$ error field.

Improved error field correction does enable operation down to lower NBI torque (magnitude $\sim 2 \mathrm{Nm}$ ), but a new MHD instability is observed before the NBI torque can be stably lowered to zero. Figure 7 has several time traces for a torque ramp-down QH-mode discharge with optimal error field correction. The frequency spectrogram of the signals from magnetic sensors shows the EHO, with dominant toroidal mode number $n=3$, and an $n=1$ mode that is destabilized around $t=3.5 \mathrm{~s}$. The $n=1$ mode eventually locks, ending the high performance phase. Ideal MHD stability analysis finds an $n=1$ kink mode calculated to become more unstable as $q_{\min }$ approaches 1 . This is an external mode with a beta threshold, not the beta-independent internal kink that MHD stability codes find when $q_{\min }$ is at or below 1 . The beta limit is predicted to reduce to the observed experimental beta value right around when the $n=1$ mode is destabilized in the experiment.

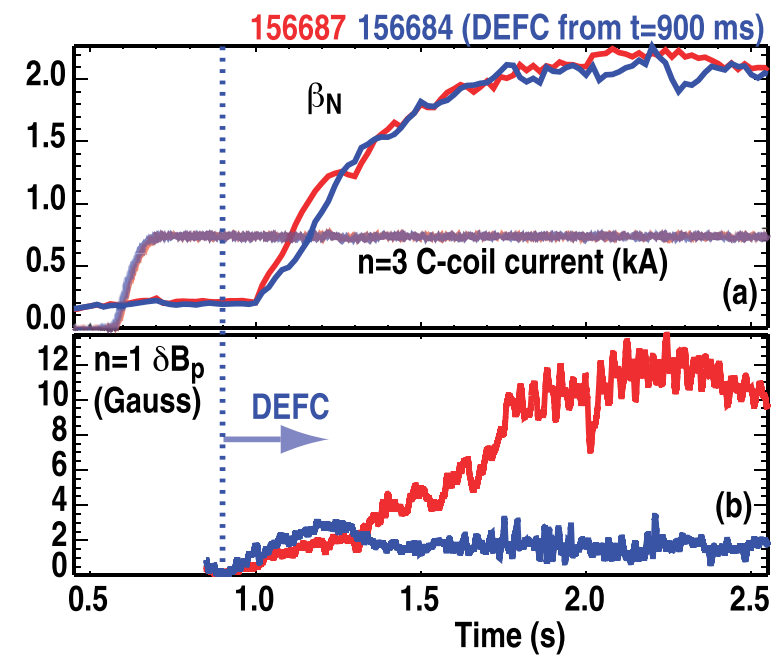

FIG. 6. Comparison of discharges with (blue traces) and without (red traces) DEFC. Time traces of (a) the normalized beta and the $n=3$ current amplitude in the C-coil; and (b) the $n=1$ poloidal field at the outer midplane. The vertical dashed line indicates when DEFC is turned on for the discharge in blue.

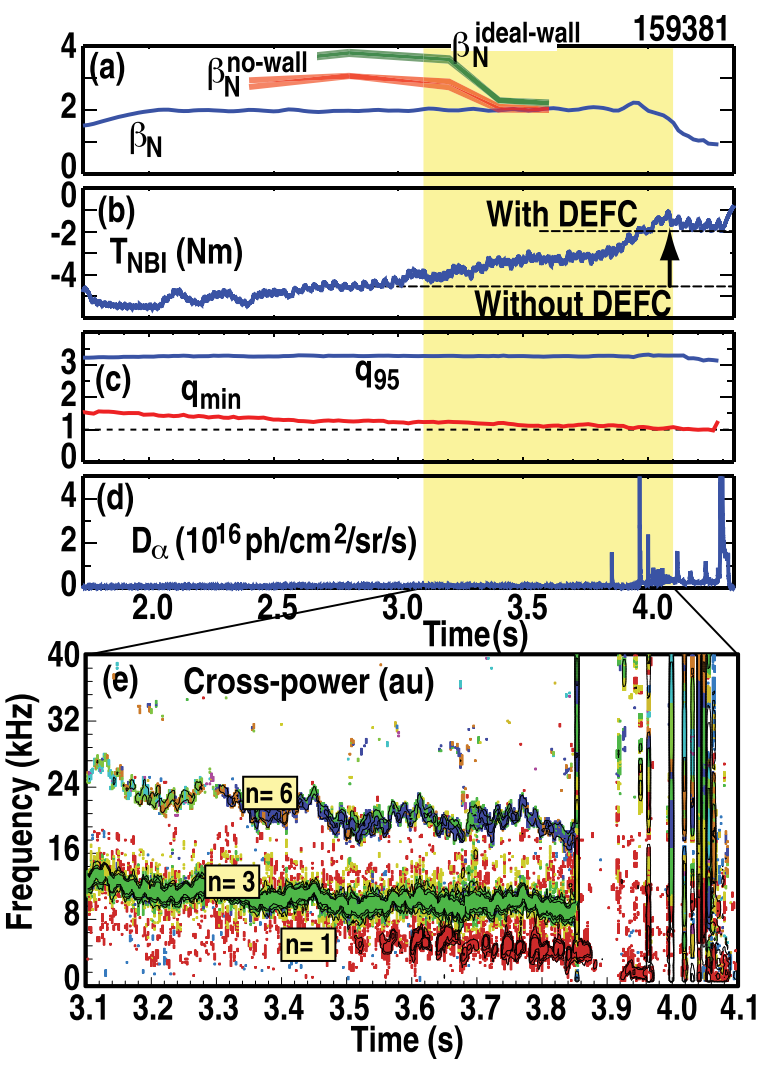

FIG. 7. Time traces of several parameters for a QH-mode discharge at ITER Baseline values of normalized beta, $\beta_{\mathrm{N}}$, and edge safety factor, $q_{95}$, with a ramp down of the NBI torque magnitude. (a) Experimental $\beta_{\mathrm{N}}$ and $\beta_{\mathrm{N}}$ stability limit predicted with an ideal wall at the position of the DIII-D vessel and with no wall. (b) NBI torque, with horizontal dashed lines indicating threshold torque against locked modes before and after implementation of DEFC. (c) Edge and minimum safety factor. (d) $\mathrm{D}_{\alpha}$ light. Yellow vertical band highlights the time range expanded in panel (e) for the spectrum of magnetic fluctuations versus frequency and time, color coded for varying toroidal mode number, $n$.

Stability analysis leads to identifying this mode as an "infernal" mode, ${ }^{11}$ that is a quasi-interchange-like mode driven by large core pressure gradient and weak magnetic shear with $q_{\min } \sim 1$. The severe impact of the infernal mode on the beta limit has been observed in many tokamaks. ${ }^{12-14}$ Figure 8 shows the equilibrium pressure and safety factor profiles, and the poloidal decomposition of the $n=1$ mode displacement, calculated by the ideal MHD stability code GATO. ${ }^{15}$ Notice the large $m=1$ component, even in the absence of a $q=1$ surface, but also the external components. Also, notice the peaked pressure and the slight negative central magnetic shear, with $q_{\min } \sim 1$.

To investigate the stability of this mode, new equilibria were constructed starting from the experimental equilibrium, and changing slightly the $q$-profile to go from NCS (experimental) to monotonic, and the pressure profile to go from peaked (experimental) to broader. All $q$-profiles have the same $q_{\min }$ and this is above 1 . All 12 combinations of $6 q$-profiles shown in Fig. 9(a) and 2 pressure profiles shown in Fig. 9(b) were analyzed. For each combination, the $\beta_{\mathrm{N}}$ stability limit is found by generating a series of new equilibria with increasing pressure at fixed $q$-profile, using the CORSICA code. ${ }^{16}$ The $\beta_{\mathrm{N}}$ stability limit calculated with no wall and with an ideal wall at the position of the DIII-D vessel is shown for each of the 12 combinations of profiles in Fig. 9(c). As the $q$ - 

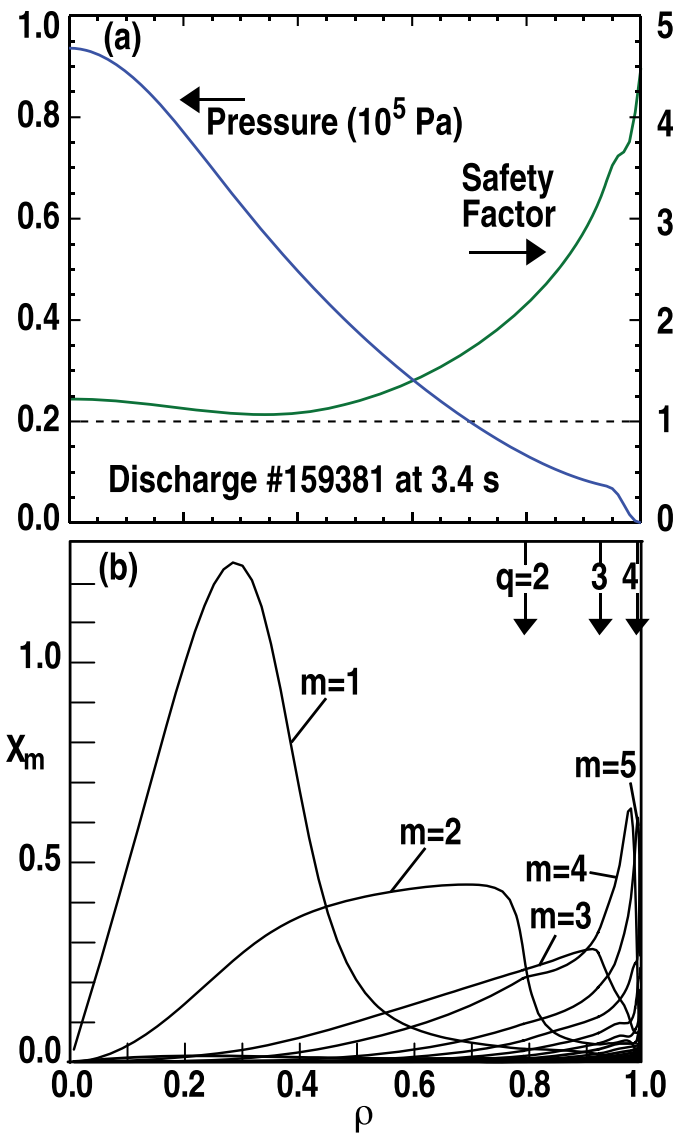

FIG. 8. (a) Radial profiles of the pressure and safety factor from kinetic reconstruction of the plasma equilibrium at a time shortly before the onset of the $n=1$ mode in Fig. 7(e). (b) Radial profiles of the poloidal harmonics of the mode perpendicular displacement, calculated by $n=1$ ideal MHD stability analysis.

profile is modified toward less NCS, the beta limit moves away from the experimental beta value. For the broader pressure profile, stability also increases with decreasing NCS, and the gain in stability from the ideal wall is stronger than for the peaked (experimental) pressure profile. These stability features are consistent with this instability being an "infernal" mode.

These studies inform the direction for future experiments: to improve the stability of the infernal mode, future experiments should try to reduce the pressure peaking, for example, by increasing density and collisionality, or by approaching $q_{\min }=1$ with a monotonic $q$-profile, rather than a weakly reversed one. In the experiments discussed in this paper, the weakly reversed central magnetic shear is due to the plasma formation approach that uses strong counter- $I_{\mathrm{p}}$ NBI during the L-mode phase, to enable robust QH-mode access. Forming the plasma with strong counter-current drive in the core tends to create a hollow current density profile. In order to avoid the NCS $q$-profile, a plasma formation approach using balanced or slightly co- $I_{\mathrm{p}}$ NBI should be adopted. This is also a necessary step toward a plasma formation approach that is relevant for ITER.

\section{DEVELOPMENT OF QH-MODE OPERATION AT HIGH DENSITY}

Until recently, the highest density QH-modes on DIII-D were at relatively low Greenwald fractions $\left(f_{\mathrm{G}} \leq 0.6\right)$
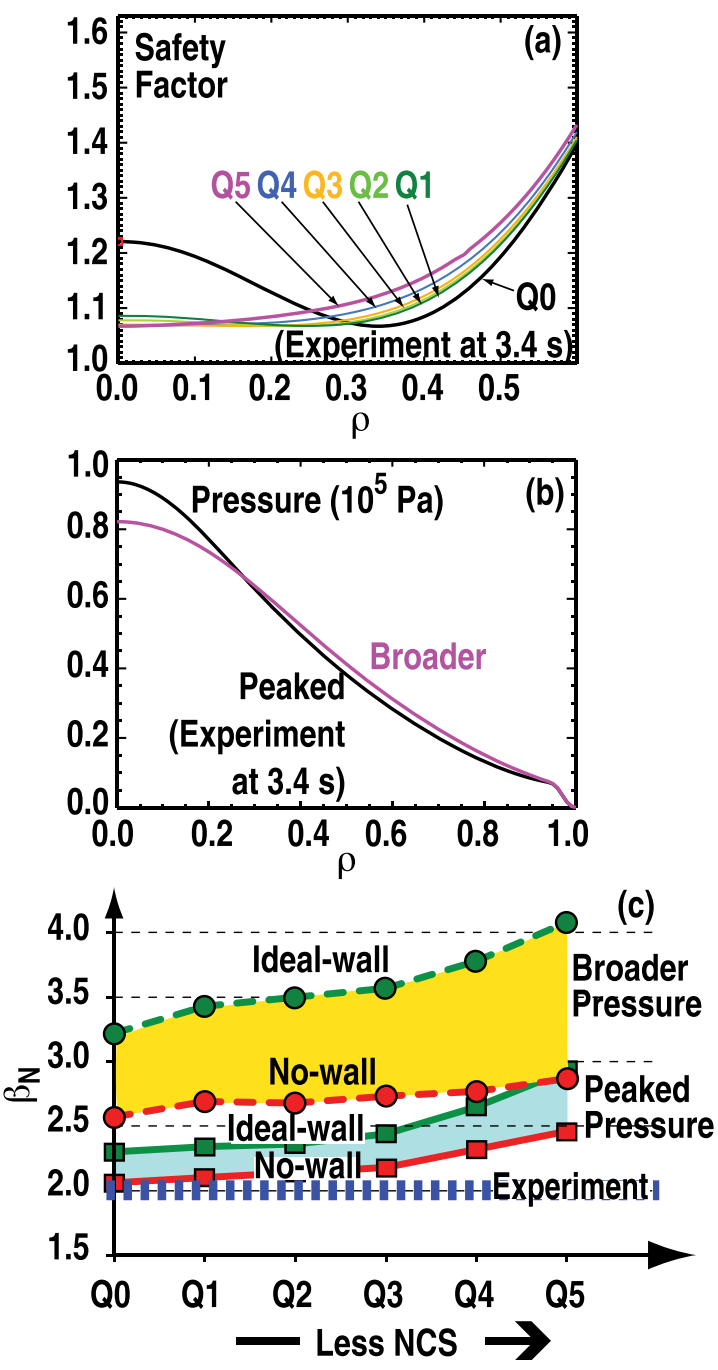

FIG. 9. (a) Series of safety factor profiles with decreasing negative central magnetic shear, constructed from the experimental equilibrium reconstruction of Fig. 8 (experimental $q$-profile is labeled Q0). All $q$-profiles are essentially identical for minor radius $\rho>0.6$. (b) Set of two pressure profiles: peaked (experimental) and broader pressure. (c) Ideal MHD $n=1$ stability limit in normalized beta obtained with no wall and with ideal wall at position of DIII-D vessel for the equilibria constructed with all combinations of safety factor and pressure profiles in (a) and (b). Horizontal dashed blue line marks the experimental value of normalized beta.

compared with ITER Baseline $\left(f_{\mathrm{G}} \sim 0.85\right)$. Here, $f_{\mathrm{G}}=n_{\mathrm{e}} / n_{\mathrm{G}}$, where $n_{\mathrm{G}}=I_{\mathrm{p}} / \pi a^{2}$ is the Greenwald density $\operatorname{limit}^{17}$ for plasma current $I_{\mathrm{p}}$ in MA and minor radius $a$ in $\mathrm{m}$. To improve the confidence that QH-mode can be accessed in ITER at high Greenwald fraction, recent experiments have investigated the physics of the QH-mode density threshold. In order to better understand the results, it is useful to first discuss the working theoretical model of QH-mode and the EHO. Over many years, DIII-D experiments have shown that the operating point of QH-mode discharges is always near but below the low $\mathrm{n}$ kink-peeling mode stability boundary in the standard H-mode pedestal stability diagram of edge current versus edge pressure gradient, shown in Fig. $10 .{ }^{18}$ One feature of the kink-peeling boundary is that rotation shear is destabilizing for modes along kink-peeling boundary. ${ }^{19}$ This has led to the hypothesis that the EHO is a nonlinearly saturated kink-peeling mode: in the presence of high edge rotation shear, a kink-peeling modes is destabilized before the edge operating point reaches the 


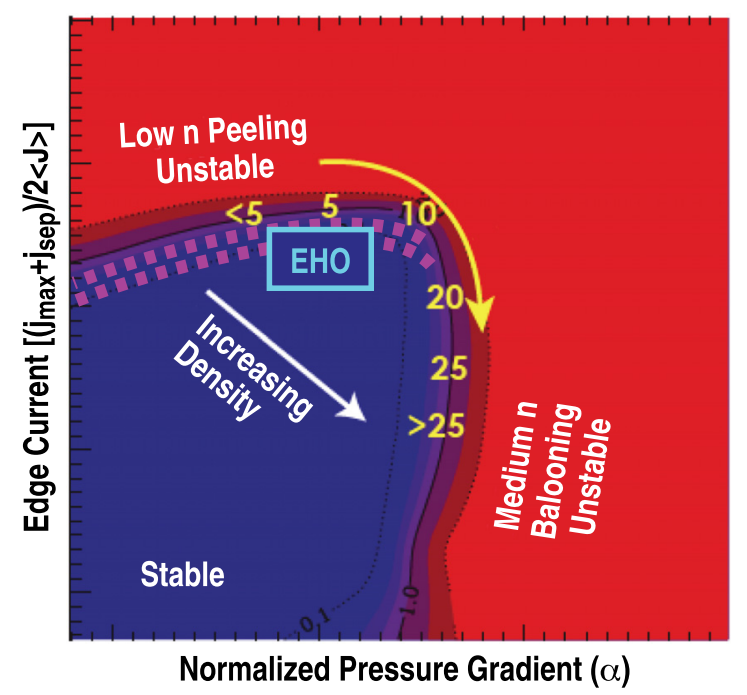

FIG. 10. Typical H-mode pedestal stability diagram versus edge current and edge pressure gradient. Blue and red indicate stable and unstable regions assuming no rotation. Operation is delimited by a boundary to low- $n$ kink peeling modes at lower density, and a boundary to medium $\mathrm{n}$ ballooning modes at higher density. The dashed purple lines sketch out the destabilizing effect on the low- $n$ peeling modes of edge rotation shear. The EHO is usually observed near but below the low- $n$ kink-peeling boundary.

no-rotation boundary, and can then saturate as the mode growth reduces rotational shear. Mode-driven transport also reduces other drive mechanisms such as pressure gradient and bootstrap current. This transport may also contribute to the nonlinear saturation of the kink-peeling mode, leading to the EHO. Typically, on DIII-D, operation near kinkpeeling boundary is obtained at low density. However, P-B theory predicts that plasma cross-section shaping strongly affects the H-mode pedestal stability space. ${ }^{19}$ The stability space is predicted to expand with triangularity, enabling operation near kink-peeling boundary at higher density and pedestal pressure. As a consequence, with weak cross section shaping QH-mode may be accessible only at low density while, with stronger shaping, QH-mode may be accessible at higher density (Fig. 4 in Ref. 19).

Previous experiments had already shown that increased plasma cross-section shaping enabled higher density QHmode. ${ }^{20}$ However, those experiments did not use gas puffing and so could not increase the density enough to reach the high Greenwald fraction of ITER. The decision not to use gas puffing in previous highly shaped discharges is historical: a consequence of the low-density threshold for $\mathrm{QH}-$ mode in weakly shaped plasmas. In the recent experiments, gas puffing was used to probe upper limits of $\mathrm{QH}$-mode operation in two different plasma cross-section shapes. ${ }^{21,22}$ At high triangularity, it was found that $\mathrm{QH}$-mode operation is compatible with high density and high Greenwald fraction. Once the EHO is established, the QH-mode edge can be robust against strong gas puffing. Figure 11 shows that the QH-mode density was raised up to $f_{\mathrm{GR}} \geq 0.8$, significantly above usual operating point, thus demonstrating that low density is not a requirement of $\mathrm{QH}$-mode operation.

These experiments also confirm a strong dependence of the QH-mode density threshold on triangularity. Figure 12 shows a summary of results from experiments with different plasma triangularity and different plasma current, at constant

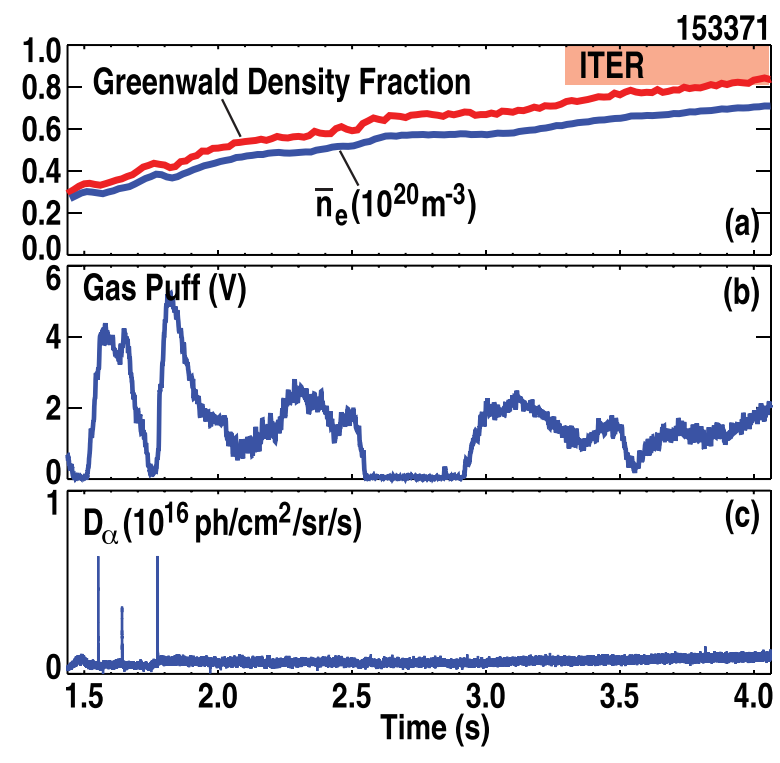

FIG. 11. Example of high density QH-mode discharge. Time traces of (a) line-averaged density and density normalized to the Greenwald limit, reaching into the ITER Baseline target range; (b) deuterium gas puff; (c) $\mathrm{D}_{\alpha}$ light.

toroidal field. For each plasma current, plasmas with strong shaping and high triangularity maintain $\mathrm{QH}$-mode at about twice the density of plasmas with reduced triangularity. Thus, the working model of the EHO based on P-B stability theory of the H-mode pedestal successfully predicts the behavior of the $\mathrm{QH}$-mode density threshold versus cross section shaping.

In addition, P-B theory predicts that, at high density and strong shaping, the pedestal height can have multiple solutions. ${ }^{23}$ Figure 13 shows the theoretically predicted pedestal height versus the pedestal density. These are results from the pedestal stability code EPED, ${ }^{24}$ which predicts pedestal height and width with no free parameters from the intersection of kinetic ballooning and P-B stability boundaries. At low triangularity $\delta=0$, peeling and ballooning modes are fairly coupled. Increasing the triangularity decouples the peeling from ballooning modes, eventually opening a valley in pedestal stability at high density. Here, there are two pedestal height (and width) solutions. The higher solution is what has been called Super H-mode. ${ }^{25}$ Constant density trajectories of increasing pedestal height (dashed line 1) lead to the usual H-Mode solution. The Super H-mode regime can

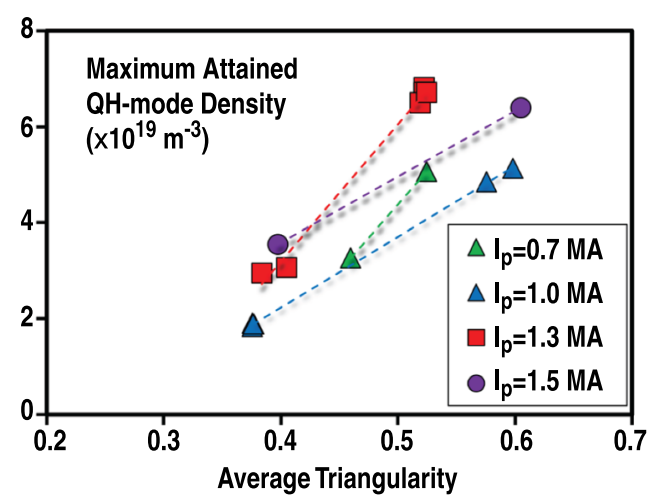

FIG. 12. Maximum attained QH-mode density versus the average triangularity of the plasma cross-section for different plasma current values. 


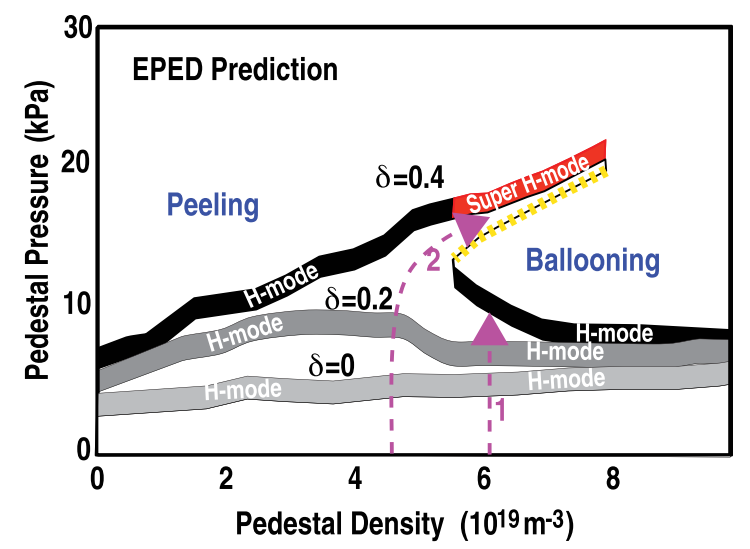

FIG. 13. EPED predictions of the pedestal height versus pedestal density for different plasma triangularities. Black and grey are the standard H-mode solutions. Red is the Super H-mode solution, only obtained at high density and high enough triangularity. Dashed yellow is a third solution found at high density and high triangularity, believed to be dynamically unstable. Dashed magenta lines are two operational trajectories discussed in the main text.

be reached with sufficient triangularity by an optimization of the pedestal height versus density trajectory (dashed line 2), that is, by first establishing H-mode at lower density, and then increasing density along the P-B boundary. The Super $\mathrm{H}$-mode regime should exist regardless whether the plasma edge is ELMing or QH-mode; QH-mode, however, is an ideal vehicle to navigate the Super H-mode stability valley, since a large ELM may send the pedestal below the lower stability boundary.

It is during the investigation of the density limit for $\mathrm{QH}-$ mode operation that the first demonstration of Super H-mode access on DIII-D was obtained. ${ }^{21}$ By simultaneously injecting gas and increasing the NBI power, both pedestal density and pressure are increased, as shown in Fig. 14. The pedestal pressure reaches very high absolute values $\sim 20 \mathrm{kPa}$ with pedestal density $\sim 8 \times 10^{19} \mathrm{~m}^{-3}$. Eventually ELMs return, and after a couple of large ELMs, the pedestal bifurcates to a state with similar density but lower pressure, demonstrating that indeed at high density there are two solutions for the
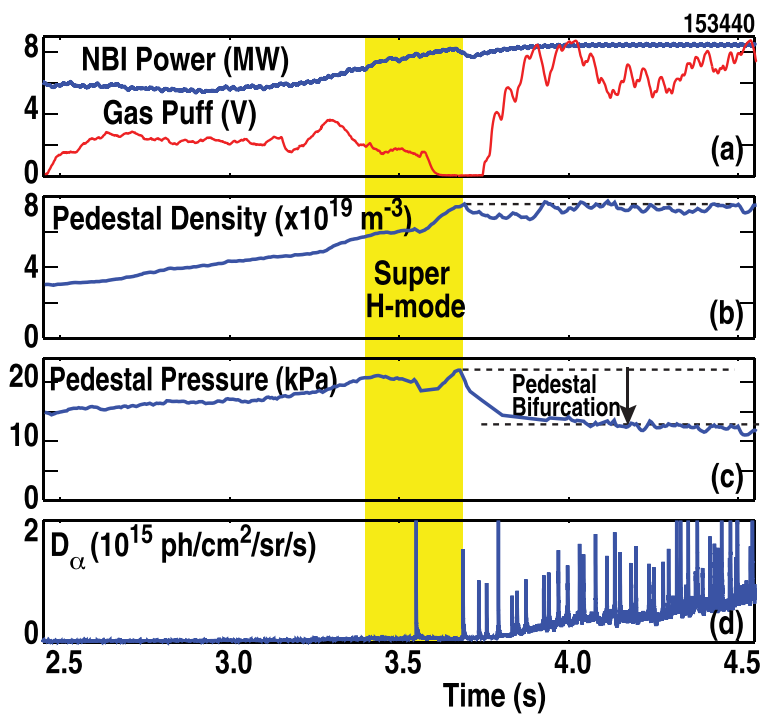

FIG. 14. Access to Super H-mode in QH-mode, and bifurcation to standard ELMing H-mode. Time traces of (a) NBI power and injected gas; (b) pedestal density; (c) pedestal pressure; (d) $\mathrm{D}_{\alpha}$ light. pedestal height: the Super H-mode solution and the lower pressure standard H-mode solution. Note that according to the EPED predictions (Fig. 13), with increasing density the Super H-mode solution can attain a pressure about double that of standard H-mode for the same density. Very high pedestal pressure should enable both high confinement and higher beta limit (broader profiles), leading to high fusion performance. Indeed, these experiments achieved $\beta_{\mathrm{N}} \sim 3$, the highest $\beta_{\mathrm{N}}$ for $\mathrm{QH}$-mode edge operation. ${ }^{22}$

\section{DEVELOPING A PREDICTIVE UNDERSTANDING OF QH-MODE FOR ITER OPERATIONS}

The experiments discussed in Sec. III have shown that, consistent with P-B theory predictions, access to $\mathrm{QH}$-mode is not limited to low Greenwald fraction, and that collisionality, via its effect on the bootstrap current and thus on the pedestal stability, is a key parameter for $\mathrm{QH}$-mode access. At low collisionality, the pedestal can operate on the P-B boundary, where QH-mode can be accessed. Also, the experimental observation of the theoretically predicted Super Hmode regime is a remarkable validation of the P-B theory embodied in the code EPED. Applied to ITER, EPED stability calculations predict that the ITER pedestal will be in the QH-mode parameter range of beta and collisionality. ${ }^{26}$ Furthermore, the calculations predict access to Super Hmode for ITER's target triangularity. ${ }^{25}$ Figure 15 shows that for nominal target density $\left(1 \times 10^{20} \mathrm{~m}^{-3}\right)$ and a range of possible $Z_{\text {eff }}$, the H-mode pedestal in ITER plasmas will operate on the kink-peeling boundary, where QH-mode can exist. With access to the Super H-mode regime, ITER could continue to operate on the kink-peeling boundary (which allows QH-mode) at significantly higher density and pedestal height than the present design target, with possible advantages for the fusion performance.

Since the EHO is postulated to be a saturated kinkpeeling mode, nonlinear simulations of the pedestal stability are required to model QH-mode. Recently, the JOREK code has been used to investigate the EHO nature ${ }^{27-29}$ in DIII-D plasmas, with the goal of later applying the code to simulations of QH-mode in ITER. JOREK is a nonlinear resistive MHD code that has been used extensively to elucidate the

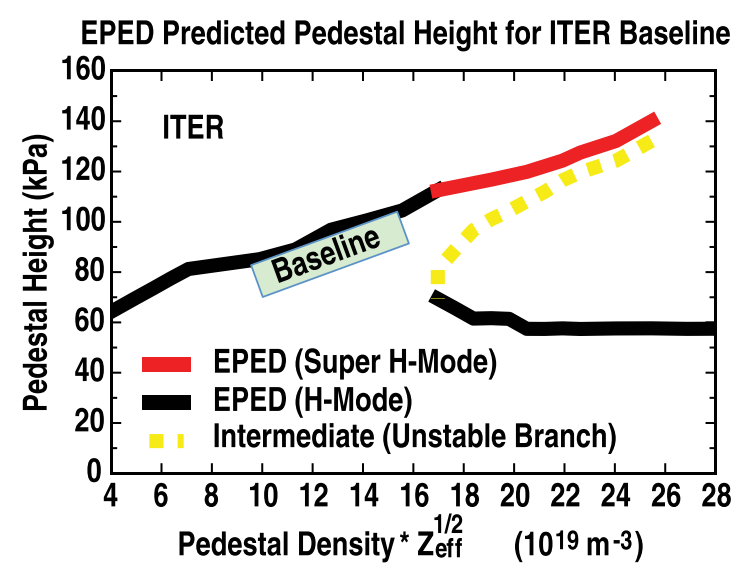

FIG. 15. EPED predictions of the pedestal height versus product of pedestal density and square root of $Z_{\mathrm{eff}}$ for the parameters of ITER Baseline. The box labeled "Baseline" spans the predicted pedestal height for ITER Baseline target pedestal density and $Z_{\mathrm{eff}}$ from 1 to 2.5 . 

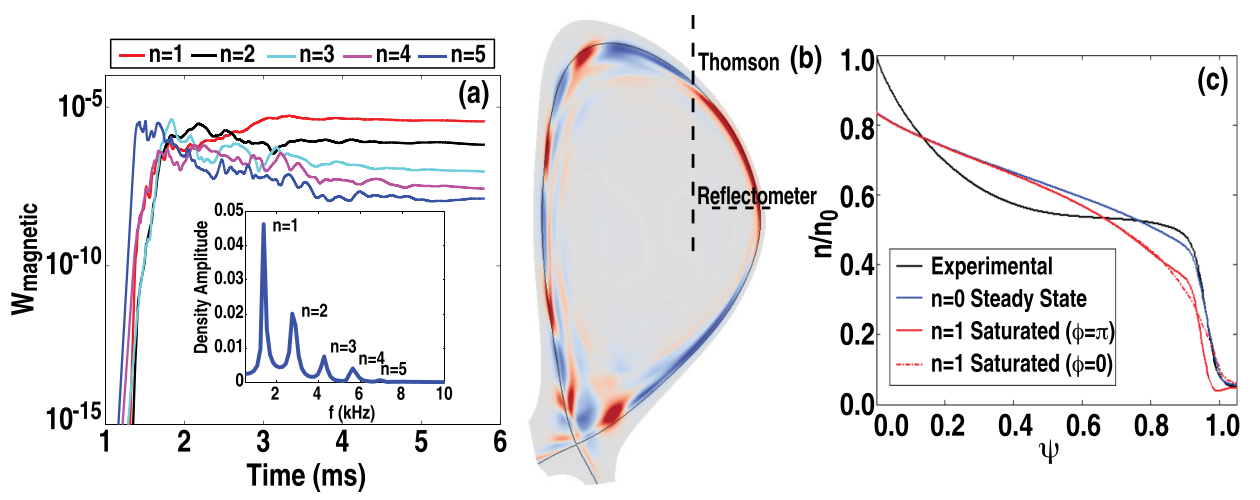

FIG. 16. JOREK stability modeling of a DIII-D QH-mode edge. (a) Time behavior of different low-n modes, showing growth and saturation over several milliseconds, and frequency spectrum of the resultant density oscillations (inset). (b) Poloidal flux perturbation from $n=1-5$ modes in the saturated state, with overlaid the measurement directions of the Thomson and Reflectometer diagnostics. (c) Experimental, JOREK initial equilibrium $(n=0)$, and JOREK perturbed density profiles from $n=1-5$ modes.

behavior of the H-mode edge. JOREK uses a reduced MHD model with realistic X-point configuration in general toroidal geometry. The code solves the visco-resistive MHD equations including diffusive particle and heat transport to evolve in time the poloidal flux, vorticity, density, and temperature, with options to include parallel rotation, and ideal wall or realistic resistive wall boundary conditions outside the separatrix. ${ }^{30}$ When utilized for nonlinear simulations of DIII-D QH-mode plasmas, JOREK calculations show low-n kinkpeeling modes growing to a saturated level, consistent with the working hypothesis on the EHO nature. An example is shown in Fig. 16. In the JOREK simulations discussed here, the initial state is the kinetic equilibrium reconstruction of a DIII-D QH-mode discharges (\#145117) during a phase with ITER-relevant NBI torque and relatively high density. The radial profiles of the particle and energy diffusivities are chosen so that the JOREK equilibrium $(n=0)$ profiles approximately match the experimental profiles when the out-flowing density and heat are compensated by the central heat and particle source described by a simple model. These particle and energy diffusivities are then assumed constant during the non-linear evolution of the instabilities.

Figure 16(a) shows the time behavior of different low-n modes over several milliseconds, and Fig. 16(b) shows the resultant perturbation of the poloidal flux in the saturated state. Note that, although higher $n$ modes are linearly more unstable, the nonlinear evolution leads to a saturated final state with lower $n$ kink-peeling modes $(n=1)$ most unstable. This is consistent with the observation that the EHO is often dominated by the $n=1$ component. Furthermore, JOREK finds that the linearly independent modes become locked in phase resulting in one non-sinusoidal oscillation containing multiple toroidal harmonics, illustrated by the inset in Fig. 16(a) with the frequency spectrum of predicted density oscillations. Qualitatively, this is indeed a typical measured EHO spectrum. The frequency of the simulation is about a factor two lower than typically measured because these simulations did not include toroidal rotation. The real frequency of the kink-peeling modes results here from the $E \times B$ flows from the nonaxisymmetric fields of the modes themselves. The addition of a parallel rotation profile, as an approximation of the experimental toroidal rotation, in general does not change the results significantly. These results are in contrast with the original hypothesis on the EHO nature conjecturing that a kink peeling mode would saturate because of the mode growth reducing the destabilizing effect of rotational shear. Saturation in the JOREK simulations is likely the result of density transport and/or the breaking of magnetic flux surfaces just inside the separatrix (discussed later in this section). These and other hypotheses are currently being investigated.

As the instability grows, transport is enhanced; therefore, the equilibrium profiles evolve away from the initial state [Fig. 16(c)]. This means that the final state of these JOREK simulations cannot be compared directly to the experimental $\mathrm{QH}$-mode used for initial input. In a different approach, the particle and energy diffusivities could be determined such that the final state of the nonlinear simulation matches the input experimental profiles. To accomplish this would require iteratively running many non-linear simulations, adjusting the diffusivities after each run until the desired $n=0$ state is attained. This however would be a very time consuming approach that may not converge, and it is reserved for future work. Since the initial experimental equilibrium is a relatively high density QH-mode, and the predicted saturated state is a lower density equilibrium, comparisons to experimental observations are carried out with respect to typical low density QH-mode plasmas, and are intended as mostly qualitative at this stage.

The density perturbations resulting from the saturated kink-peeling modes in JOREK have been compared to measurements in QH-mode plasmas with $n=1$ dominated EHO. The simulated perturbations reproduce characteristics typical of EHO measurements. Figure 17 compares JOREK simulations with density oscillations measured by the DIII-D microwave reflectometer. The scanning frequency microwave reflectometer ${ }^{31}$ can measure the radial density profile along the outboard midplane [as shown in Fig. 16(b)] with high time resolution of up to $\sim 25$ microseconds, sufficient to resolve the oscillation caused by the EHO rotating past the diagnostic at $\sim 4 \mathrm{kHz}$. The figure compares edge profiles at three phases of the density oscillation corresponding to minimum, intermediate, and maximum perturbed poloidal field measured by fast magnetic probes. Both measurements and simulations show a near-rigid displacement of the density profile (note the similar displacement magnitude), suggesting that the perturbation may be due to a rotating kink-like 

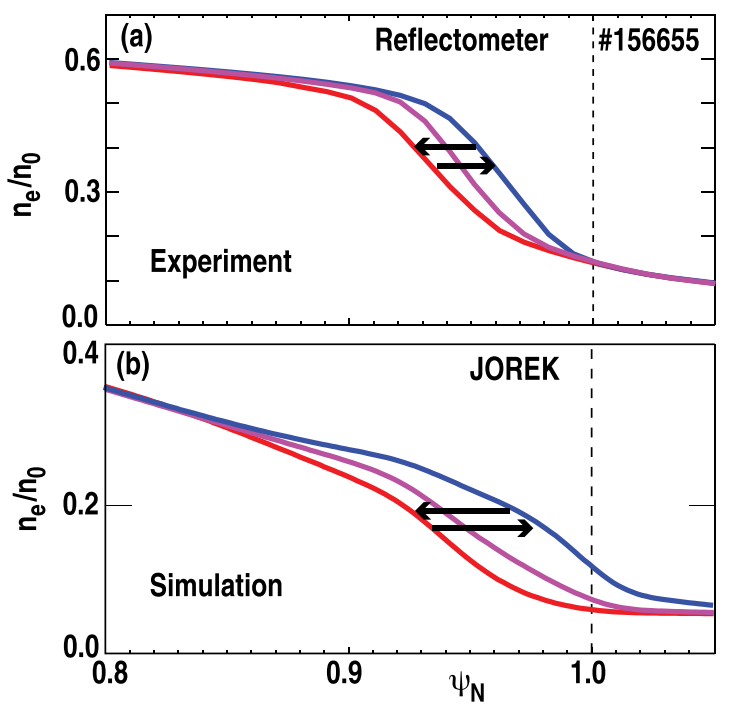

FIG. 17. Edge density profile oscillation at outer midplane from (a) $n=1$ dominated EHO measured by microwave reflectometer; and (b) saturated kink-peeling modes $(n=1-5)$ in JOREK simulation.

structure. However, further comparisons with JOREK results suggest that this kink perturbation also generates a significant ergodic region at the edge of the plasma. Comparing the density profile time averaged over one or more cycles of the oscillation reveals a flattening of the profile just inside the separatrix. Figure 18 shows a comparison between the simulated profile and the profile measured by the Thomson scattering diagnostic. The flattening is more evident in the Thomson data, likely because of more flux expansion along the Thomson view [also shown in Fig. 16(b)], but it is also observed on the time averaged reflectometer data. The flat spot in the time-averaged density profile is also reproduced in the JOREK simulations of the saturated kink-peeling
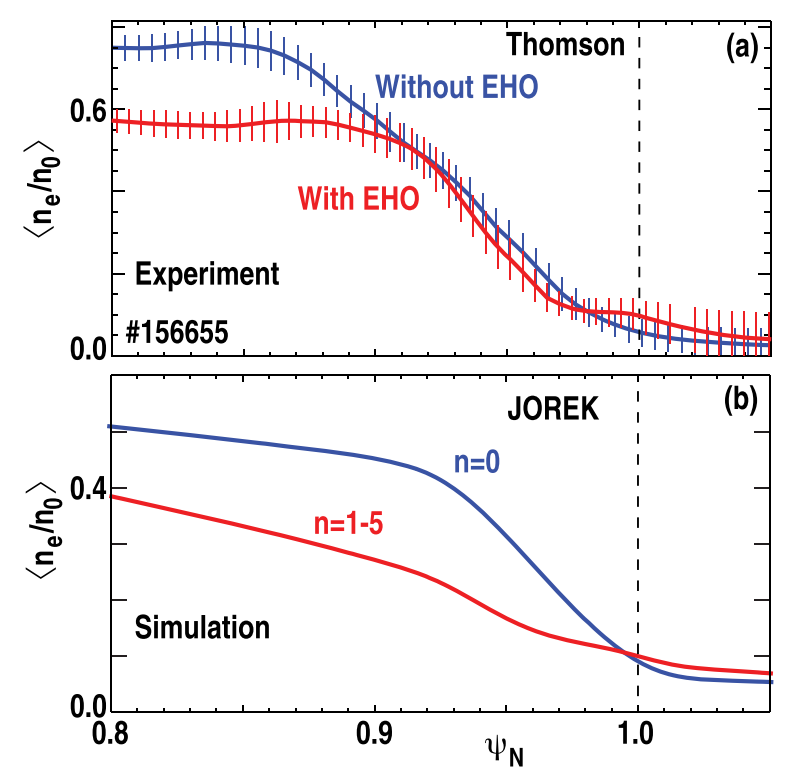

FIG. 18. Comparison of measured and simulated edge density profiles along the Thomson scattering view. (a) Time averaged profiles from Thomson scattering data in two phases of the same discharge: without coherent EHO and with $n=1$ dominated EHO. (b) JOREK initial equilibrium profile $(n=0)$, and JOREK with time averaged saturated kink-peeling modes $(n=1-5)$. modes. An understanding of this flat spot may be drawn out of the Poincare section of field lines in the poloidal plane near the X-point shown in Fig. 19. Notice how the magnetic perturbation of the saturated kink modes leads to a significant ergodic region already for $\rho>0.8$, with some small, embedded remnant islands. However, only outside $\rho \sim 0.95$, the field lines are poorly confined (large radial excursions and short connection length to the wall). This region of strong ergodic transport is consistent with the location of the flat spot observed in the density profiles. Unlike a single magnetic island, an ergodic layer is nearly axisymmetric, thus it can result in a flat spot in the time averaged profiles. The formation of an ergodic region in the plasma edge may play a key role in the kink-peeling mode saturation leading to the EHO in QH-mode plasmas. Further work is needed to understand the precise saturation mechanisms. More details on the effect of ergodization on the energy and particle transport in the JOREK non-linear simulations of these DIII-D QH-mode plasmas can be found in Ref. 29.

\section{QH-MODE PARTICLE TRANSPORT}

A main motivation of $\mathrm{QH}$-mode research has been the prospects of eliminating the pulsed ELM power fluxes to the PFCs in a fusion reactor. However, ELMs also carry out the essential function of expelling impurities from the core of the plasma. Impurities are unavoidable in a fusion reactor, for example, helium is an inherent impurity because of the fusion reaction. A buildup of impurities in the core would cool the plasma and dilute the density of the fusion fuel, thus reducing the fusion performance. It is therefore essential to evaluate the impact on impurity transport of replacing an ELMing edge with a QH-mode edge in a fusion plasma.

Building on previous work, ${ }^{20}$ recent experiments have demonstrated that particle transport in $\mathrm{QH}$-mode can be fast enough for impurity exhaust. In these experiments, a fluorine impurity was injected (as carbon tetrafluoride, $\mathrm{CF}_{4}$ ) in plasmas of similar density, but different types of $\mathrm{H}$-mode edge: ELMing, RMP ELM-suppressed, and QH-mode. ${ }^{32}$ Fluorine was chosen because it is not normally present in the tokamak environment (non-intrinsic) and it is highly reactive, so that once it exits the plasma core it does not re-enter (non

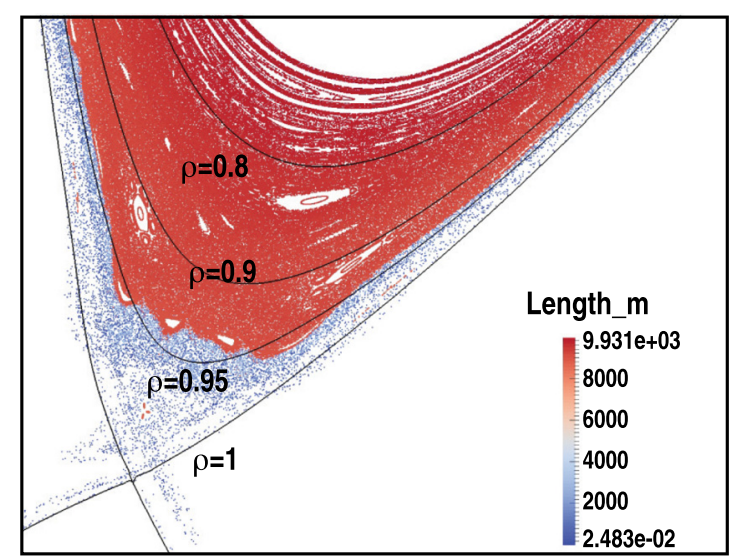

FIG. 19. Poincaré section of magnetic field lines near the divertor in the JOREK simulation with saturated kink-peeling modes $(n=1-5)$. The length of the field lines before they intercept the wall is color coded. 


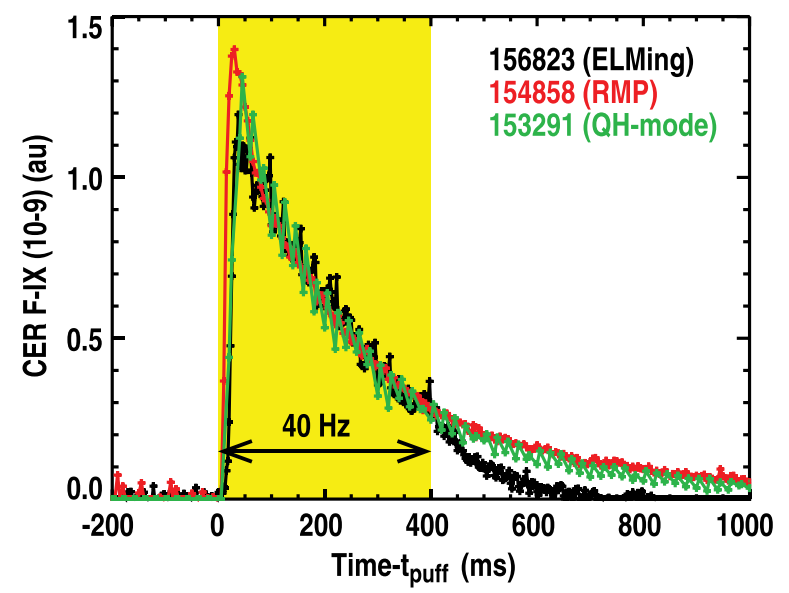

FIG. 20. Decay of the beam-induced charge-exchange emission from the FIX (10-9) transition at $4796 \AA$, after fluorine injection and uptake in the plasma core. An exponential decay character is observed after the peak intensity in discharges without ELMs (QH-mode and RMP ELM-suppressed) and during constant ELM frequency (here $40 \mathrm{~Hz}$ ) in an ELMing discharge. Correspondent impurity confinement time is approximately $335 \mathrm{~ms}$. In the ELMing discharge, the ELM frequency increases after $400 \mathrm{~ms}$ and the confinement time becomes shorter.

recycling). Without impurity sources other than the controlled gas puff, the impurity confinement time, $\tau_{\mathrm{p}}$, can be evaluated from the simple decay of the fluorine chargeexchange emission intensity following a short duration injection. ${ }^{33}$ Figure 20 shows that the impurity exhaust rate measured in QH-mode is the same as that measured in ELMing discharge with $40 \mathrm{~Hz}$ ELMs and in RMP ELM-suppressed discharge, all at similar density. Notice that for ELMing discharges, the impurity exhaust is faster for higher ELM frequency. The $n=1$ dominated EHO in QH-mode provides much better impurity exhaust than observed in the ITER Baseline Scenario on DIII-D with lower frequency ELM $(\sim 10 \mathrm{~Hz})$. This is also a typical ELM frequency for high current operation in JET.

The EHO driven impurity exhaust is found to be insensitive to the toroidal rotation. It does, however, depend on the equilibrium density, and the magnitude of the EHO amplitude. ${ }^{32}$ Results of the dependence on the atomic number of the impurity ion are being analyzed.

\section{SUMMARY}

Through a coordinated work of numerical simulations and DIII-D experiments, significant progress has been made in advancing the QH-mode operating space and physics basis, increasing confidence that QH-mode will enable high confinement, ELM-stable operation on ITER. For the first time, QH-mode has been achieved and maintained for many energy confinement times in an ITER-like shape at values of beta, confinement, and safety factor that together extrapolate to $Q=10$ operation in the ITER baseline scenario. Extension to ITER-equivalent NBI torque and plasma rotation presents challenges that are peculiar to the condition with parameters of $\beta_{\mathrm{N}}, \mathrm{H}_{98 \mathrm{y} 2}$, and $q_{95}$ simultaneously matching the ITER Baseline targets. At those parameters, good error field correction is critical because plasma amplification of a residual $n=1$ error field can be significant. The magnetic feedback based technique of dynamic error field correction, previously only used at plasma beta above the no-wall MHD stability limit, has been successfully extended to the moderate beta of the ITER baseline scenario. However, an "infernal" mode is observed, caused by the strong counter NBI formation scenario adopted in experiments so far. Progress toward a more ITER relevant scenario includes developing a formation scenario using balanced injection, which should also ameliorate the infernal mode stability.

Also for the first time, QH-mode operation has been extended to ITER-relevant values of normalized density $\sim 80 \%$ of the Greenwald limit, overcoming the long-standing low-density limit of QH-mode operation. This has been achieved by tailoring the plasma shape to improve the edge stability as predicted by the P-B stability theory of the Hmode pedestal. Importantly, these experiments have shown that access to QH-mode is not correlated with low Greenwald fraction, and that collisionality (via its effect on bootstrap current) is the relevant density parameter. The P-B theory as embodied in the code EPED also predicted that at high density a new H-mode edge regime should be accessible with strong shaping of the plasma cross section, a regime called Super H-mode because it is characterized by about twice the edge pedestal pressure possible in standard $\mathrm{H}$ mode at the same high pedestal density. The DIII-D results are in excellent agreement with these predictions, providing a new successful benchmarking of the code EPED. Applied to ITER, EPED predicts that the ITER edge will operate on the pedestal stability boundary where QH-mode can exist. Integration of high density QH-mode operation with ITER Baseline fusion performance now appears as an attainable goal for future experiments.

For the first time, nonlinear MHD simulations of a $\mathrm{QH}-$ mode edge plasma have resulted in the prediction of a multiharmonic edge oscillation consistent with the experimentally observed EHO. These calculations, carried out using the code JOREK, confirm the hypothesis that the EHO consists of saturated kink-peeling modes. The JOREK results and comparisons with new measurements (taking advantage of the improved Thomson scattering diagnostic) have also yielded the first evidence that these saturated modes lead to an ergodic layer at the plasma edge, just inside the separatrix. While more accurate measurements and simulations are desirable, these results represent new and essential steps on the path to predictive modeling of the EHO and its effect on the edge particle transport.

Finally, recent experiments have developed a technique to compare impurity transport rates during different $\mathrm{H}$-mode edge regimes, and show that $\mathrm{QH}$-mode particle transport can be fast enough for impurity exhaust without ELMs. Analysis of the $\mathrm{Z}$ dependence of the $\mathrm{EHO}$ particle transport is ongoing.

\section{ACKNOWLEDGMENTS}

This material is based upon work supported by the U.S. Department of Energy, Office of Science, Office of Fusion Energy Sciences, using the DIII-D National Fusion Facility, a DOE Office of Science user facility, under Award Nos. DE-FC02-04ER54698, DE-AC02-09CH11466, DE-FG02-07ER54917, DE-FG02-04ER54761, and DE- 
FG02-08ER54984, and the ITER Organization. The views and opinions expressed herein do not necessarily reflect those of the ITER Organization. DIII-D data shown in this paper can be obtained in digital format by following the links at https://fusion.gat.com/global/D3D_DMP.

${ }^{1}$ K. H. Burrell, M. E. Austin, D. P. Brennan, J. C. DeBoo, E. J. Doyle, P. Gohil, C. M. Greenfield, R. J. Groebner, L. L. Lao, T. C. Luce, M. A. Makowski, G. R. McKee, R. A. Moyer, T. H. Osborne, M. Porkolab, T. L. Rhodes, J. C. Rost, M. J. Schaffer, B. W. Stallard, E. J. Strait, M. R. Wade, G. Wang, J. G. Watkins, W. P. West, and L. Zeng, Plasma Phys. Controlled Fusion 44, A253 (2002).

${ }^{2}$ ITER Physics Basis, Nucl. Fusion 47, S1 (2007).

${ }^{3}$ ITER Physics Groups on Confinement and Transport and Confinement Modeling and Database, ITER Physics Basis Editors, and ITER EDA, Nucl. Fusion 39, 2175 (1999).

${ }^{4}$ C. Angioni, A. G. Peeters, F. Jenko, and T. Dannert, Phys. Plasmas 12, 112310 (2005).

${ }^{5}$ A. R. Polevoi, M. Shimada, M. Sugihara, Yu. L. Igitkhanov, V. S. Mukhovatov, A. S. Kukushkin, S. Yu. Medvedev, A. V. Zvonkov, and A. A. Ivanov, Nucl. Fusion 45, 1451 (2005).

${ }^{6}$ A. M. Garofalo, W. M. Solomon, J.-K. Park, K. H. Burrell, J. C. DeBoo, M. J. Lanctot, G. R. McKee, H. Reimerdes, L. Schmitz, M. J. Schaffer, and P. B. Snyder, Nucl. Fusion 51, 083018 (2011).

${ }^{7}$ C. Paz-Soldan, T. C. Luce, A. M. Garofalo, G. L. Jackson, R. J. La Haye, J. M. Hanson, K. E. J. Olofsson, F. Turco, B. A. Grierson, and W. M. Solomon, Bull. Am. Phys. Soc. 59, 327 (2014).

${ }^{8}$ A. M. Garofalo, K. H. Burrell, W. M. Solomon, M. E. Fenstermacher, J. M. Hanson, M. J. Lanctot, M. Okabayashi, P. B. Snyder, and DIII-D Team, in Proceedings of the 39th European Physical Society Conf. on Plasma Physics (Stockholm, Sweden, 2012), see http://ocs.ciemat.es/ epsicpp2012pap/pdf/O2.102.pdf.

${ }^{9}$ K. H. Burrell, A. M. Garofalo, W. M. Solomon, M. E. Fenstermacher, D. M. Orlov, T. H. Osborne, J.-K. Park, and P B. Snyder, Nucl. Fusion 53, 073038 (2013).

${ }^{10}$ A. M. Garofalo, E. J. Strait, L. C. Johnson, R. J. La Haye, E. A. Lazarus, G. A. Navratil, M. Okabayashi, J. T. Scoville, T. S. Taylor, and A. D. Turnbull, Phys. Rev. Lett. 89, 235001 (2002).

${ }^{11} \mathrm{~J}$. Manickam, T. Fuijita, N. Gorelenkov, A. Isayama, Y. Kamada, M. Okabayashi, M. Bell, R. Bell, R. Budny, E. Fredrickson, S. Ishida, Y. Ishii, F. Leventon, T. Ozeki, S. Tkeji, and M. Zarnstorff, Nucl. Fusion 39, 1819 (1999).

${ }^{12}$ T. Ozeki, M. Azumi, Y. Kamada, S. Ishida, Y. Neyatani, and S. Tokuda, Nucl. Fusion 35, 861 (1995).

${ }^{13}$ S. Günter, A. Gude, J. Hobirk, M. Maraschek, S. Saarelma, S. Schade, R. C. Wolfe, and ASDEX Upgrade Team, Nucl. Fusion 41, 1283 (2001).

${ }^{14}$ A. Martynov, PhD dissertation, see http://infoscience.epfl.ch/record/33686 (2005).

${ }^{15}$ L. C. Bernard, F. J. Helton, and R. W. Moore, Comput. Phys. Commun. 24, 377 (1981).

${ }^{16}$ J. A. Crotinger, L. L. LoDestro, L. D. Pearlstein, A. Tarditi, T. A. Casper, and E. B. Hooper, Lawrence Livermore National Laboratory Report No. UCRIL-ID-126284, March 19, 1997. See National Technical Information Service Document PB2005-102154. Copies may be ordered from the National Technical Information Service, Springfield, VA 22161.

${ }^{17}$ M. Greenwald, Plasma Phys. Controlled Fusion 44, R27 (2002).
${ }^{18}$ T. H. Osborne, P. B. Snyder, K. H. Burrell, T. E. Evans, M. E. Fenstermacher, A. W. Leonard, R. A. Moyer, M. J. Schaffer, and W. P. West, J. Phys.: Conf. Ser. 123, 012014 (2008).

${ }^{19}$ P. B. Snyder, K. H. Burrell, H. R. Wilon, M. S. Chu, M. E. Fenstermacher, A. W. Leonard, R. A. Moyer, T. H. Osborne, M. Umansky, W. P. West, and X. Q. Xu, Nucl. Fusion 47, 961 (2007).

${ }^{20}$ K. H. Burrell, W. P. West, E. J. Doyle, M. E. Austin, T. A. Casper, P. Gohil, C. M. Greenfield, R. J. Groebner, A. W. Hyatt, R. J. Jayakumar, D. H. Kaplan, L. L. Lao, A. W. Leonard, M. A. Makowski, G. R. McKee, T. H. Osborne, P. B. Snyder, W. M. Solomon, D. M. Thomas, T. L. Rhodes, E. J. Strait, M. R. Wade, G. Wang, and L. Zeng, Phys. Plasmas 12, 056121 (2005).

${ }^{21}$ W. M. Solomon, P. B. Snyder, K. H. Burrell, M. E. Fenstermacher, A. M. Garofalo, B. A. Grierson, A. Loarte, G. R. McKee, R. Nazikian, and T. H. Osborne, Phys. Rev. Lett. 113, 135001 (2014).

${ }^{22}$ W. M. Solomon, K. H. Burrell, M. E. Fenstermacher, A. M. Garofalo, B. A. Grierson, A. Loarte, G. R. McKee, R. Nazikian, T. H. Osborne, and P. B. Snyder, "Extending the physics basis of quiescent H-mode toward ITER relevant parameters," Nucl. Fusion (submitted).

${ }^{23}$ P. B. Snyder, T. H. Osborne, K. H. Burrell, J. Candy, R. J. Groebner, A. W. Leonard, R. Nazikian, D. M. Orlov, O. Schmitz, M. R. Wade, and H. R. Wilson, "The EPED pedestal model: extensions, application to elmsuppressed regimes, and ITER predictions," in Proceedings of the 24th IAEA Fusion Energy Conf., San Diego, California, 2012 (IAEA, Vienna), CD-ROM file th_p3-17.pdf and http://www-naweb.iaea.org/napc/physics/ FEC/FEC2012/html/fec12.htm.

${ }^{24}$ P. B. Snyder, R. J. Groebner, A. W. Leonard, T. H. Osborne, and H. R. Wilson, Phys. Plasmas 16, 056118 (2009).

${ }^{25}$ P. B. Snyder, W. M. Solomon, K. H. Burrell, A. M. Garofalo, B. A. Grierson, R. J. Groebner, A. W. Leonard, R. Nazikian, T. H. Osborne, E. A. Belli, J. Candy, and H. R. Wilson, "Super H-mode: Theoretical prediction and initial observations of a new high performance regime for tokamak operation," Nucl. Fusion (submitted).

${ }^{26}$ K. H. Burrell, A. M. Garofalo, W. M. Solomon, M. E. Fenstermacher, T. H. Osborne, J.-K. Park, M. J. Schaffer, and P. B. Snyder, Phys. Plasmas 19, 056117 (2012).

${ }^{27}$ G. T. A. Huijsmans, F. Liu, S. Futatani, A. Lorate, F. Köchl, M. Hoelzl, A. M. Garofalo, and E. Nardon, "Non-Linear MHD simulations for ITER," in Proceedings of the 25th IAEA Fusion Energy Conference, St. Petersburg, Russia, Paper TH/6-1Ra, see http://www-pub.iaea.org/MTCD/Meetings/ PDFplus/2014/cn221/cn221ConferenceProgrammeAndAbstracts.pdf.

${ }^{28}$ F. Liu, G. T. A. Huijsmans, A. Lorate, A. M. Garofalo, W. M. Solomon, P. B. Snyder, and M. Hoelzl, "Nonlinear MHD simulations of QH-mode plasmas in DIII-D," in Proceedings of the 41st EPS Conference on Plasma Physics, Berlin, Germany (2014).

${ }^{29}$ F. Liu, G. T. A. Huijsmans, A. Loarte, A. M. Garofalo, W. M. Solomon, P. B. Snyder, and M. Hoelzl, "Nonlinear MHD simulations of Quiescent Hmode plasmas in DIII-D," Nucl. Fusion (submitted).

${ }^{30}$ G. T. A. Huijsmans, S. Pamela, E. Van der Plas, and P. Ramet, Plasma Phys. Controlled Fusion 51, 124012 (2009).

${ }^{31}$ L. Zeng, W. A. Peebles, E. J. Doyle, T. L. Rhodes, N. Crocker, X. Nguyen, C. W. Wannberg, and G. Wang, Rev. Sci. Instrum. 85, 11D843 (2014).

${ }^{32}$ B. A. Grierson, K. H. Burrell, R. M. Nazikian, W. M. Solomon, A. M. Garofalo, E. A. Belli, G. M. Staebler, M. E. Fenstermacher, G. R. McKee, T. E. Evans, D. M. Orlov, S. P. Smith, C. Chrobak, C. Chrystal, and DIIID Team, Phys. Plasmas 22, 055901 (2015).

${ }^{33}$ B. A. Grierson, K. H. Burrell, A. M. Garofalo, W. M. Solomon, A. Diallo, and M. O'Mullane, Nucl. Fusion 54, 114011 (2014). 